\title{
Evaluation of Cost Functions for Gray Value Matching of 2D Images in Radiotherapy
}

\author{
Niels Dekker, Lennert S. Ploeger, and Marcel van Herk \\ Radiotherapy Department, The Netherlands Cancer Institute / Antoni van Leeuwenhoek \\ hospital, Plesmanlaan 121, 1066 CX Amsterdam, The Netherlands \\ \{ndekker, lennert, portal\}@nki.nl
}

\begin{abstract}
In this paper, cost functions are tested for 2D registration of portal and reference images for treatment verification in radiotherapy. Tests were performed with and without pre-processing for 96 image pairs of various treatment sites. The best results were obtained when the images were preprocessed by unsharp masking and histogram equalization. This led to a success rate of about $88 \%$ and a difference with clinical registration of $1.2 \mathrm{~mm} \mathrm{SD}$ and 1 degree SD for most of the tested cost functions. The number of local minima with mutual information is much larger than with the other tested cost functions.
\end{abstract}

\section{Introduction}

External beam radiotherapy is often verified by electronic portal imaging, imaging the patient with the treatment beam. As part of the image analysis to determine the setup error, the anatomy in a portal image is matched with the anatomy in a reference image. As reference images, often diagnostic x-ray images or digitally reconstructed radiographs are used. Because of the complexity of 3D-2D matching, 2D image analysis is still used on a wide scale. Most automatic matching algorithms for portal images are based on segmented anatomy. These methods are especially successful for images of the pelvic region. The purpose of this study is to find a cost function that is applicable for anatomy matching on a wide range of treatment sites.

\section{Material and Methods}

In this study 96 pairs of portal and reference images are used. The images were selected at random from rectum, salivary gland, whole brain, prostate and lung treatments. For each tumor site, 9 or 10 anterior-posterior and 9 or 10 lateral image pairs were used. Each of the portal images was matched previously in the clinic. These clinical matches are considered "ground truth".

It appeared that the result of the cost functions could be improved by applying two pre-processing steps to both the portal and the reference image: unsharp masking followed by histogram equalization of the region within the field edge mask. 
The investigated cost functions are computed from a cross-histogram of pixel values of both images. The following cost functions were tested: 1. Mutual information (MI); 2. The root mean square of the pixelwise differences (RMS); 3. The negated mean pixelwise product (PROD), which is similar to image correlation; and 4. The mean absolute pixelwise difference (DIFF).

For each matched pair of portal and reference images the cost function values were calculated for more than 20,000 transformations; these transformations were generated by varying the translation up to $\pm 1 \mathrm{~cm}$ in horizontal and vertical direction from the clinical match and by varying the rotation up to \pm 10 degrees. This search range corresponds with the range of expected setup errors in clinical practice. The step size is $0.7 \mathrm{~mm}$ for translation and 1 degree for rotation. The transformation that corresponds to the global minimum of the cost function values is determined. If this transformation lies within a sphere around the clinical match of $4 \mathrm{~mm}$ translation and 4 degrees rotation, the match is considered successful. For each cost function the number of successes is counted, and the average and the standard deviation of the results of the successful matches is computed to determine the accuracy compared with the ground truth.

\section{Results}

Table 1 gives an overview of the successful matches of the tested cost functions. MI had 470 local minima; the other cost functions had 13 to 17 local minima on average.

Table 1. Overview of the performance of the cost functions. The success rate and the mean (standard deviation) of the difference with the clinical match are presented. In all cases, unsharp masking followed by histogram equalization was used as preprocessing.

\begin{tabular}{lllll}
\hline & MI & RMS & PROD & DIFF \\
\hline Success rate & $78 \%$ & $89 \%$ & $88 \%$ & $89 \%$ \\
Horizontal translation $(\mathrm{cm})$ & $0.01(0.10)$ & $0.01(0.10)$ & $-0.01(0.10)$ & $0.01(0.10)$ \\
Vertical translation $(\mathrm{cm})$ & $0.00(0.11)$ & $-0.02(0.12)$ & $-0.02(0.12)$ & $-0.02(0.12)$ \\
Rotation (degrees) & $-0.05(0.93)$ & $0.02(1.02)$ & $-0.18(1.01)$ & $-0.01(0.98)$ \\
\hline
\end{tabular}

\section{Discussion and Conclusions}

The success rate is reasonable, if one considers that the existing registration method has a poor success rate for difficult cases like the lung and lateral pelvis images. Good results were obtained for most treatment sites. The largest number of failures occurred for small brain fields, where little anatomical detail is visible. The difference in success rate between the different costs functions is small when pre-processing is applied. In conclusion, gray value matching of portal and reference images is feasible and forms a useful alternative for methods based on segmented anatomy. 